\title{
The burden of urological disease in Zomba, Malawi: A needs assessment in a sub-Saharan tertiary care center
}

Tristan Juvet ${ }^{1}$; James R. Hayes ${ }^{2}$; Sarah Ferrara ${ }^{1}$; Duncan Goche ${ }^{3}$; Robert D. Macmillan ${ }^{4}$; Rajiv K. Singal ${ }^{5}$

${ }^{1}$ Division of Urology, University of Toronto, Toronto, ON, Canada; ${ }^{2}$ Division of Urology, University of Ottawa, Ottawa, ON, Canada; ${ }^{3}$ Department of Surgery, Zomba Central Hospital, Zomba, Malawi; ${ }^{4}$ Division of Urology, Kelowna General Hospital, Kelowna, BC, Canada; ${ }^{5}$ Division of Urology, Michael Garron Hospital, Toronto, ON, Canada

Cite as: Can Urol Assoc J 2019 June 17; Epub ahead of print. http://dx.doi.org/10.5489/cuaj.5837

Published online June 17, 2019

$* * *$

\section{Abstract}

Introduction: A large part of the developing world continues to lack access to surgical care. Urology remains one of the least represented surgical subspecialties in global health. To begin understanding the burden of urological illness in sub-Saharan Africa, we sought to characterize all patients presenting to a tertiary care hospital in Malawi with a urological diagnosis or related complaint in the past year.

Methods: Retrospective review of the surgical clinic and surgical theater record books at Zomba Central Hospital ( $\mathrm{ZCH}$ ) was performed over a one-year time span. Patients presenting with urological diagnoses or undergoing a urological procedure under local or general anesthetic in the operating theater were identified and entered into a database.

Results: A total of 440 clinical patients were reviewed. The most common clinical presentations were for urinary retention (34.7\%) and lower urinary tract symptoms (15.5\%); 182 surgical cases were reviewed. The most common diagnoses for surgical patients were urethral stricture disease (22\%), bladder masses (17\%), and benign prostatic hyperplasia (BPH) symptoms (14.8\%). Urethral stricture-related procedures, including direct visual internal urethrotomy and urethral dilatation were the most common (14.2\% and 7.7\%). BPH-related procedures, including simple prostatectomy and transurethral resection of the prostate were the second most common $(6.7 \%$ and $8.2 \%)$.

Conclusions: Urethral stricture disease, $\mathrm{BPH}$, and urinary retention represent the clinical diagnoses with the highest burden of visits. Despite these numbers, few definitive procedures are performed annually. Further focus on urological training in sub-Saharan Africa should focus on these conditions and their surgical management. 


\section{Introduction}

A large part of the developing world continues to lack access to surgical care. This situation is accentuated in sub-Saharan Africa. Over the past decades, surgical care has been recognized as an important aspect of global health, benefitting from increased funding and a higher frequency of international missions. ${ }^{1}$ Despite these advances, urology remains one of the least represented surgical subspecialties in global health with expenditures well below most other fields. ${ }^{2}$ Global surgery missions are seldom focused on urologic care alone.

The American Urology Association (AUA) has partnered with International Volunteers in Urology (IVUmed), one of the few urology-focused non-governmental organizations, and certainly the largest. This partnership provides funding for volunteer missions incorporating both American and Canadian urologists, resident scholarships and local training programs. The Canadian Urological Association provides regular scholarship programs to provide training for urologists practicing in developing countries. The European Association of Urology supports various African urology programs through joint meetings and courses.

In 2002, a charter of “urologic rights” was drafted by Dr. Neville Harrison. This outlined the basic treatments to which patients across the world should have access. This list included treatment of urinary retention, hematuria, urethral stricture disease, urethral trauma, vesicovaginal fistula, penile carcinoma and access to male circumcision. The most common illnesses thought to be encountered by practicing urologists in sub-Saharan Africa include benign prostatic hyperplasia (BPH), urethral stricture disease, prostate cancer, bladder cancer and urethral/ureteric trauma. ${ }^{3}$

Untreated urologic conditions represent a major burden on patients in the developing word. Previous studies have found that urologic diseases were responsible for over 25 million disability-adjusted life years worldwide, with the highest overall rates in sub-Saharan Africa. ${ }^{4}$ While there is minimal data available on urologic surgical rates, surgical volumes in general are known to be lowest in the developing world. In a review of worldwide surgical volumes, it was noted that the poorest third of the world's population received only 3.5\% of worldwide surgical operations. ${ }^{5}$ Studies from Sierra Leone have estimated that a quarter of the previous year's deaths among regular households could have been prevented with timely surgical management. ${ }^{6}$ Further compounding these issues is the lack of trained staff and equipment. The majority of subSaharan countries have access to only a handful of urologists. The most populous country in Africa, Nigeria, has one urologist per 3.2 million people. Training programs are uncommon and funding for both education and access to care is limited. ${ }^{7}$ There are approximately 145000 physicians in sub-Saharan Africa for a population over one billion. ${ }^{8}$ In addition, urology remains a specialty where a large amount of specialized equipment requiring regular maintenance is needed.

Zomba is Malawi's fourth largest city with a regional population of over 600000 . Zomba Central Hospital $(\mathrm{ZCH})$ is one of the four national tertiary care centers with a referral population of approximately four million. ${ }^{9} \mathrm{ZCH}$ is unique in Malawi as it is one of the only sites offering 
urologic procedures. Additional surgical services at the site include general surgery, orthopedic surgery and obstetrics and gynecology.

Care at ZCH, as in many sub-Saharan clinics and hospitals, is mainly provided by clinical officers: non-physicians who have undergone approximately two to three years of generalist post-secondary training. Malawian clinical officers undergo an additional one year internship following their training. Along with nurse practitioners, physician's assistants and similar professions, they are categorized as non-physician clinicians (NPCs). In Malawi there are over twenty times more NPCs than there are physicians. ${ }^{10}$ With appropriate training and supervision, surgical NPCs were found to have complication rates similar to surgeons at $\mathrm{ZCH}^{9}{ }^{9}$

Prevalence studies on urologic diseases in this region of the world remain limited in quantity. This in turn may limit the resources provided to particular areas in terms of equipment, funding and appropriately trained staff. In order to begin understanding the burden of urological illness in this community, we sought to characterize all patients presenting to the surgical clinic with a urologic diagnosis or related complaint in the past year.

\section{Methods}

Retrospective review of the surgical clinic and surgical theater record books at Zomba Central Hospital (ZCH) was performed for a one year time span from March 2017 to March 2018 and manually transcribed into an electronic database. This review was performed as part of an expanding global surgery initiative within the Division of Urology at the University of Toronto. We examined the clinical records of all patients presenting to the surgical clinic with a urologic diagnosis or chief complaint. Clinical visit diagnoses were based on presenting symptoms or known previous medical history as assessed by the clinical staff. Similarly, for surgical records, we extracted relevant data for all patients undergoing urologic procedures. This included any procedure performed by the urology clinical officer or visiting urologists under local or general anesthetic in the operating room.

As there is no computer system within the hospital, the information contained in these logbooks was manually entered. Clinical logbook data was entered by both the administrative staff and clinical officers. Surgical logbooks were filled by clinical officers and surgeons, both local and visiting.

Information was taken from the patient's "Health Passport”; a small handwritten booklet owned by the patient containing all relevant medical information as documented by medical personnel. Data obtained from clinical records included age, gender, diagnosis and month of service. Data from surgical logs included age, gender, diagnosis, procedure and anaesthetic (local, spinal, or general).

The majority of operating room urologic procedures were performed by the trained urology clinical officer. In certain cases, assistance was sought from the single attending general surgeon present at the hospital. In other circumstances visiting international surgeons from Canada or Germany assisted with procedures. Urologic operative cases were included based on 
procedure and diagnosis and the completion of the procedure by the general surgery team did not exclude it from this study.

\section{Results}

Review and analysis of the surgical clinic record revealed a total of 440 patients visited the surgical clinic for a urology-specific complaint over the one year time frame (Table 1). 425 (96.59\%) patients were male. The most common complaints were related to urinary retention (34.77\%), lower urinary tract symptoms (15.45\%), hydrocele (10.23\%), urethral stricture (8.86\%) and bladder mass (6.82\%). It should be noted that retention related visits included both acute and chronic presentations. Additional common diagnoses included orchitis/scrotal pain (6.14\%) and phimosis (4.32\%).

The median age of patients attending the surgical clinic with a urologic complaint was 48 years-old. Men presenting to the clinic with urinary retention were older and had a mean age of 71.1 years. Patients presenting with urethral stricture were younger and on average were 46.7 years old. The youngest patients were those presenting with phimosis related complaints with an average age of 17 years. One three year old patient was seen for an undescended testicle.

Review of surgical records showed 182 urologic cases performed over the one-year time frame (Table 2.1). Median age of surgical patients was 50 years. The most common diagnosis for patients undergoing surgery was urethral stricture disease (21.98\%), followed by bladder mass (17.03\%), BPH (14.84\%), hydrocele (9.34\%), and prostate cancer (5.49\%). Of the recorded cases, the most common procedure performed was cystoscopy (30 cases, 16.48\%), followed by direct visual internal urethrotomy (DVIU) (26 cases, 14.29\%), hydrocelectomy (17 cases, 9.34\%), simple prostatectomy (15, 8.24\%), urethral dilatations (14 cases, $7.69 \%)$, and transurethral resections of the prostate (TURP) (12 cases, 6.59\%). (Table 2.2)

Of note, only a single urethrocutaneostomy was performed over the course of this year for penile trauma. No urethroplasties or urethral reconstructive surgeries were performed for stricture disease. Two penectomies were performed in conjunction with a visiting surgeon from Germany. Of the four radical cystectomies performed, three were with assistance from the attending Malawian general surgeon. Diversion techniques were not recorded. A single radical nephrectomy was also performed by the general surgeon. Surgical assists are summarized in Table 2.2.

Spinal anesthesia was used in 104 (68.7\%) cases and was the preferred option by the anesthetists and clinical officers at ZCH. Larger and longer procedures were performed under a general anesthetic (29 cases, 15.93\%). Shorter less invasive procedures such as cystoscopy and urethral dilation were mainly performed with local anesthetic alone (28 cases, 15.38\%). (Table 2.3)

\section{Discussion}

This study shows a diverse presentation of urologic conditions, both clinically and surgically, in a large urban center with no formally trained practicing urologist. Urinary retention-related 
complaints made up over a third of our clinic visits, yet represented only 27 surgical cases (14.83\%). Anecdotally, an estimated 2000 regional patients live with indwelling catheters. Adding to the issue, catheters are often placed in rural clinics without a clear diagnosis or follow up plan.

Furthermore, access to equipment and training for endoscopic management of BPH remains highly challenging. Open simple prostatectomies remain commonly performed in Malawi by both clinical officers and general surgeons, however more urgent surgical procedures are often prioritized. Most TURPs performed at ZCH were in conjunction with visiting international surgeons due to lack of training and experience with the resectoscope.

Household survey data from a study in Sierra Leone identified a prevalence of $1.99 \%$ for men with urinary retention and $2.18 \%$ percent for men with gross hematuria. Over half the men in this study had retention for over a year and approximately $20 \%$ reported not being able to work due to this condition. Furthermore, $48 \%$ could not afford treatment or surgical care. ${ }^{11}$ Interestingly, gross hematuria was not identified as a presenting complaint amongst our patients, although it may represent a subset of patients presenting with lower urinary tract symptoms (LUTS).

Visits for LUTS ranged around 15\% at our site, similar to previous reports showing a prevalence of about $19 \%$ in East African men. ${ }^{12}$ A recent assessment of the prevalence of LUTS in Ugandan men above the age of 55 found rates of $40.5 \%$ for moderate symptoms and $20 \%$ for severe symptoms as per the International Prostate Symptom Scale. ${ }^{13}$ Extrapolating this data to encompass sub-Saharan Africa, the authors estimate that over 20 million men suffer from moderate to severe LUTS.

Although the severity of LUTS was not quantified in our review, previous studies from Ghana and Uganda have shown that it is uncommon for men to present with mild to moderate lower urinary tract symptoms and surgery is often performed only in cases of retention. ${ }^{14}$ Among men presenting to a Ugandan urology clinic for LUTS, 82.4\% were experiencing moderate to severe LUTS. These patients expressed lower quality of life, increased bother from their symptoms and experienced daily worry and work interference associated with their symptoms. ${ }^{15}$ A 2010 review of worldwide LUTS found that over 1.1 billion people worldwide were affected by LUTS in 2008, with Africa expecting the biggest increase in affected individuals over the next decade. ${ }^{16}$

These numbers highlight a greater need for sustainable surgical management of urinary retention and recalcitrant LUTS. In addition, further measures are needed to promote public awareness and educate our local colleagues that LUTS are amenable to simple and inexpensive medical treatment when a timely diagnosis is made. Ignorance with regards to symptom progression and late presentation currently contribute to presentation with advanced disease, unacceptable morbidity and the need for surgical intervention.

Cystoscopy aside, DVIUs represented the second most common urologic surgical procedure at $\mathrm{ZCH}$ and fourth most common clinical visit. Small studies from Cameroon and 
Nigeria have yielded similar data with regards to average age groups (mean age between 40 and 60 years of age) and initial treatments consisting primarily of suprapubic diversion and urethral dilatation and/or DVIU. Over half of all strictures were attributed to sexually transmitted infections in these reviews. ${ }^{17,18}$ Geographical differences in stricture etiology have been studied, with trauma and idiopathic causes being predominant in the developing world. ${ }^{19}$

Many patients presenting with stricture disease at ZCH are often seen with recurrent or lengthy strictures. These patients would be considered poor candidates for repeated VIU and/or dilatations within our healthcare system and would ideally best be served by urethroplasty. We propose that perineal urethrostomy would be an ideal approach for many of these patients, requiring the least resources and training. Costs for a formal urethroplasty in a similarly sized Nigerian hospital were reported to be 800-1000 USD\$, well out of the means of most subSaharan Africans. Lack of surgical training in this field among local practitioners remains a major barrier to treatment of urethral stricture disease in this region.

Treatment of malignancies in sub-Saharan Africa is uncommon and patients often present late in the course of their disease. Prostate cancer incidence is thought to be rising in many African countries, although the exact number remains difficult to quantify due to lack of proper registries. ${ }^{20}$ Prostate cancer is thought to be the most common malignancy among men in subSaharan Africa and it is often underdiagnosed and/or underreported. ${ }^{21}$ A review of Nigerian patients with prostate cancer found that over $94 \%$ presented with advanced disease. Similarly to our patients, bilateral simple subcapsular orchiectomy was the preferred treatment option. ${ }^{22}$ Antiandrogens, access to radiotherapy and chemotherapy are scarcely available and expensive. ${ }^{23}$ General knowledge surrounding prostate cancer and availability of PSA screening have been shown to be lacking in a similar population in a large urban center in Uganda. ${ }^{24}$

Data on bladder cancer incidence and treatment in Africa remains extremely limited. Epidemiological studies have shown that incidence rates in African men and women remain some of the lowest in the world. Some exceptions were noted however, with higher rates among Egyptian men and Malawian women. ${ }^{25}$ While women only represented 10\% (18/182) of all surgical cases, 42\% (13/31) of all patients undergoing bladder mass surgeries including TURBT, radical cystectomy or partial cystectomy were women.

Schistosomiasis is thought to be a leading cause of bladder cancer in Malawi as it is in most of sub-Saharan Africa, although exact data on prevalence remains unclear. ${ }^{26,27}$ In a review of biopsies performed in Blantyre, Malawi, about 53\% of bladder biopsies were found to contain squamous cell carcinoma, and $67.4 \%$ of these patients were women. ${ }^{28}$ At our center, most patients undergoing cystoscopy appeared to have advanced disease and only young healthy patients were given an opportunity at surgical management of suspected bladder cancer, often without a definitive preoperative diagnosis. Both surgical and non-surgical patients with bladder cancer are often lost to follow-up

Access to pathology remains a significant problem in sub-Saharan Africa. A survey in 2012 identified only six licensed pathologists present within Malawi. ${ }^{29}$ This represents 
approximately one pathologist per 2.6 million people, in comparison with the US average of one per 20000 people. In our center, transportation of the specimen and costs associated with specimen processing were the responsibility of the patient and their families. The average cost of having a specimen analyzed at the nearest University hospital was estimated to be USD \$30, a fee the majority of the population cannot afford. As such, most specimens are disposed of following surgery.

Interestingly, obstetric fistula disease was not a clearly identified diagnosis in our review, despite representing a major public health issue in sub-Saharan Africa with up to 130000 new cases every year. ${ }^{30}$ Most of the fistula repairs at $\mathrm{ZCH}$ are performed by the attending gynecologist, and these are few in number. We suspect that the presence of well-established programs for fistula repair in the cities of Blantyre and Lilongwe lead most patients with this condition to seek treatment at these centers. ${ }^{31}$

An initial partnership with a Canadian urologist spearheaded the construction of new urology-focused operating rooms and donation of new endoscopic equipment. Visiting Canadian and German urologists were able to perform procedures requiring additional expertise and technical skills beyond those of the clinical associates or the on-site $\mathrm{ZCH}$ general surgeon. These procedures focused mostly on use of the resectoscope, pediatric surgery and penile surgery. While initial Canadian visits were performed under the auspices of a prominent Canadian NGO in Malawi, more recent collaborations have been through the nascent University of Toronto global surgery program.

These visits have provided valuable training to the clinical officers on site and this has translated into a higher volume of cases, improved surgical technique and more frequent use of endoscopic surgery at the site, namely DVIUs. While attempts have been made to schedule specialized and complex cases in conjunction with international visits, the lack of adequate medical records and communication means between the hospital and patients makes this challenging. Early coordination with ZCH staff and case selection is a goal of future visits. Limitations to this study include lack of more detailed diagnoses available from both the clinical and surgical logbooks and lack of additional retrospective data. Data are often entered by nurses or NPCs and patients often have little knowledge of any previous diagnoses when returning to clinic. Patients presenting after-hours or for emergency cases were also not included in the clinical logbooks. Certain diagnoses may not be accurate given the lack of laboratory or diagnostic investigations such as PSA testing or biopsy. Volume of surgical procedures at ZCH were also often limited by availability of an anesthetist or anesthesia NPC. Although we believe that these findings are relatable to other large sub-Saharan African hospitals, this is yet to be confirmed in similar studies.

\section{Conclusions}

The range of cases seen at $\mathrm{ZCH}$ are similar to previously reported data in sub-Saharan Africa. The most common presentations are for issues related to urinary retention, urethral stricture 
disease and bladder cancer. The most commonly encountered surgical procedures are related to endoscopic management of urethral stricture disease and BPH-related surgery (open simple prostatectomy or TURP). While bladder cancer is frequently encountered, treatment options are limited and patients present late in the course of their disease. Further focus on urologic training in sub-Saharan Africa should focus on these conditions and their surgical management and coordination with ongoing public health efforts to reduce the incidence of schistosomiasis. As our global urology efforts expand, we hope to continue increasing the number of visiting trainees and practicing urologists to provide both teaching and logistical support for urologic services at ZCH. A Malawian resident is also undergoing Canadian-funded training in Zambia through the College of Surgeons of East, Central and Southern Africa (COSECSA). At completion of his training in late 2019, he will be joining ZCH as its first fully trained urologist. The goal of our program is to have him establish one of the few urologic practices in this region of the world. Identifying and training future residents would have the potential to create an official COSECSA training program in Zomba. Canadian urologists can play a key role in contributing to surgical teaching in Africa and the development of recognized training programs. We hope to continue expanding this role with future visits, including an upcoming visit in June 2019 in conjunction with our colleagues from Germany. Additional plans include allocating funds for outreach camps at local district hospitals, improving access to histopathological analysis and upgrades to the operating rooms and equipment. 


\section{References}

1. Gutnik LA, Dielman J, Dare AJ, et al. Funding flows to global surgery: an analysis of contributions from the USA. Lancet 2015;385:S51.

2. Gutnik L, Yamey G, Riviello R, et al. Financial contributions to global surgery: an analysis of 160 international charitable organizations. Springerplus 2016;5:1558.

3. Campain NJ, MacDonagh RP, Mteta KA, et al. Global surgery - How much of the burden is urological? BJU Int 2015;116:314-16.

4. Murray CJL, Vos T, Lozano R, et al. Disability-adjusted life years (DALYs) for 291 diseases and injuries in 21 regions, 1990-2010: A systematic analysis for the Global Burden of Disease Study 2010. Lancet 2012;380:2197-223.

5. Weiser TG, Regenbogen SE, Thompson KD, et al. An estimation of the global volume of surgery: a modelling strategy based on available data. Lancet 2008;372:139-44.

6. Groen RS, Samai M, Stewart KA, et al. Untreated surgical conditions in Sierra Leone: A cluster randomised, cross-sectional, countrywide survey. Lancet 2012;380:1082-87.

7. Olapade-Olaopa EO, Onawola KA. Challenges for urology in sub-Saharan Africa in 2006. J Men's Heal Gend 2006;3:109-16.

8. Mullan F, Frehywot S, Omaswa F, et al. Medical schools in sub-Saharan Africa. Lancet 2011;377:1113-121.

9. Wilhelm TJ, Thawe IK, Mwatibu B, et al. Efficacy of major general surgery performed by non-physician clinicians at a central hospital in Malawi. Trop Doct 2011;41:71-5.

10. Mullan F, Frehywot S. Non-physician clinicians in 47 sub-Saharan African countries. Lancet 2007;370:2158-163.

11. Patel HD, Kamara TB, Kushner AL, et al. Gross hematuria and urinary retention among men from a nationally representative survey in sierra leone. Urology 2014;83:1273-78.

12. Chokkalingam AP, Yeboah ED, DaMarzo A, et al. Prevalence of BPH and lower urinary tract symptoms in West Africa. Prostate cancer postate Dis 2012;15:170-76.

13. Bajunirwe F, Stothers L, Berkowitz J, et al. Prevalence estimates for lower urinary tract symptom severity among men in Uganda and sub-Saharan Africa based on regional prevalence data. Can Urol Assoc J 2018;12:447-452.

14. Kyei MY, Mensah JE, Morton B, et al. Surgical management of BPH in Ghana: a need to improve access to transurethral resection of the prostate. East Afr Med J 2012;89:241-45.

15. Stothers L, MacNab AJ, Bajunirwe F, et al. Associations between the severity of obstructive lower urinary tract symptoms and care-seeking behavior in rural Africa: A cross-sectional survey from Uganda. PLoS One 2017;12:1-10.

16. Irwin DE, Kopp ZS, Agatep B, et al. Worldwide prevalence estimates of lower urinary tract symptoms , overactive bladder , urinary incontinence and bladder outlet obstruction. BJU Int 2011;108:1132-39.

17. Olajide AO, Olajide FO, Kolawole OA, et al. A retrospective evaluation of challenges in urethral stricture management in a tertiary care centre of a poor resource community. Nephrourol Mon 2013;5:974-77.

18. Ngaroua, Eloundou NJ, Djibrilla Y, et al. Aspects épidémiologiques, cliniques et prise en charge de sténose urétrale chez l'adulte dans un Hôpital de District de Ngaoundéré, Cameroun. Pan Afr Med J 2017;26:1-6.

19. Stein DM, Thum DJ, Barbagli G, et al. A geographic analysis of male urethral stricture 
aetiology and location. BJU Int 2013;112:830-34.

20. Adeloye D, David RA, Aderemi AV, et al. An estimate of the incidence of prostate cancer in Africa: A systematic review and meta-analysis. PLoS One 2016;11:1-18.

21. Rebbeck TR, Devesa SS, Chang B-L, et al. Global patterns of prostate cancer incidence, aggressiveness, and mortality in men of african descent. Prostate Cancer 2013;2013:1-12.

22. Badmus TA, Adesunkanmi ARK, Yusuf BM, et al. Burden of prostate cancer in southwestern Nigeria. Urology 2010;76:412-16.

23. Kingham TP, Alatise OI, Vanderpuye V, et al. Treatment of cancer in sub-Saharan Africa. Lancet Oncol 2013;14:e158-67.

24. Nakaandi H, Kirabo M, Semgabo C, et al. Knowledge, attitudes and practices of Ugandan men regarding prostate cancer. Afr J Urol 2013;19:165-70.

25. Babjuk M. Trends in bladder cancer incidence and mortality: Success or disappointment? Eur Urol 2017;71:109-110.

26. Makaula P, Sadalaki JR, Muula AS, et al. Schistosomiasis in Malawi: A systematic review. Parasites and Vectors 2014;7:570.

27. Bowa K, Mulele C, Kachimba J, et al. A review of bladder cancer in sub-Saharan Africa: A different disease, with a distinct presentation, assessment, and treatment. Ann Afr Med 17:99-105.

28. Mtonga P, Masamba L, Milner D, et al. Biopsy case mix and diagnostic yield at a Malawian central hospital. Malawi Med J 2013;25:62-64.

29. Adesina A, Chumba D, Nelson AM, et al. Improvement of pathology in sub-Saharan Africa. Lancet Oncol 2013;14:e152-57.

30. Wall LL. Obstetric vesicovaginal fistula as an international public-health problem. Lancet 2006;368:1201-09.

31. Drew LB, Wilkinson JP, Nundwe W, et al. Long-term outcomes for women after obstetric fistula repair in Lilongwe, Malawi: A qualitative study. BMC Pregnancy Childbirth 2016;16:1-12. 
Figures and Tables

\begin{tabular}{|c|c|c|}
\hline Diagnosis & n (\%) & Average age \\
\hline Retention & $153(34.77 \%)$ & 71.1 \\
\hline Lower urinary tract symptoms & $68(15.45 \%)$ & 70.16 \\
\hline Hydrocele & $45(10.23 \%)$ & 43.42 \\
\hline Stricture & $39(8.86 \%)$ & 46.67 \\
\hline Bladder mass & $30(6.82 \%)$ & 51.52 \\
\hline Orchitis & $27(6.14 \%)$ & 43.36 \\
\hline Phimosis & $19(4.32 \%)$ & 17.24 \\
\hline Prostate cancer & $12(2.73 \%)$ & 66.73 \\
\hline Prostatitis & $10(2.27 \%)$ & 64.22 \\
\hline Paraphimosis & $8(1.82 \%)$ & 18.25 \\
\hline Scrotal mass & $5(1.14 \%)$ & 39.2 \\
\hline Penile cancer & $3(0.68 \%)$ & 50 \\
\hline Hydronephrosis & $2(0.45 \%)$ & 54.5 \\
\hline Hypospadias & $2(0.45 \%)$ & 18 \\
\hline Incontinence & $2(0.45 \%)$ & 49.5 \\
\hline Scrotal abscess & $2(0.45 \%)$ & 50 \\
\hline Scrotal swelling & $2(0.45 \%)$ & 31 \\
\hline Bladder stone & $1(0.23 \%)$ & 72 \\
\hline Circumcision & $1(0.23 \%)$ & 24 \\
\hline Imperforate vagina & $1(0.23 \%)$ & 70 \\
\hline Kidney abscess & $1(0.23 \%)$ & 48 \\
\hline Kidney stone & $1(0.23 \%)$ & 35 \\
\hline Meatal stenosis & $1(0.23 \%)$ & 55 \\
\hline Penile ulcer & $1(0.23 \%)$ & 67 \\
\hline Postop scrotal hematoma & $1(0.23 \%)$ & 74 \\
\hline Scrotal fistula & $1(0.23 \%)$ & 46 \\
\hline UDT & $1(0.23 \%)$ & 3 \\
\hline Urethral trauma & $1(0.23 \%)$ & 41 \\
\hline Total & $440(100.00 \%)$ & 57.6 \\
\hline \multicolumn{3}{|l|}{ Clinic visits by gender } \\
\hline \multicolumn{3}{|l|}{ Male: 425 patients (96.59\%) } \\
\hline Female: 15 patients (3.41\%) & & \\
\hline
\end{tabular}


Table 2.1. Most responsible diagnosis for patients undergoing urologic surgery

Diagnosis

Stricture

Bladder mass

$\mathrm{BPH}$

Hydrocele

Prostate cancer

Necrotizing fasciitis

Testicular torsion

Penile cancer

Bladder stone

Cryptorchidism

Bladder neck stenosis

Hypospadias

Meatal stenosis

Phimosis

n (\%)

$40(21.98 \%)$

Priapism

Testicular lesion

$31(17.03 \%)$

$27(14.84 \%)$

Ambiguous genitalia

$17(9.34 \%)$

Bladder ulcers

Gross hematuria

$10(5.49 \%)$

Hydronephrosis

7 (3.85\%)

$6(3.30 \%)$

Kidney stone

$5(2.75 \%)$

Paraphimosis

$4(2.20 \%)$

Penile trauma

$4(2.20 \%)$

3 (1.65\%)

$3(1.65 \%)$

$3(1.65 \%)$

$2(1.10 \%)$

Perforated bladder

$2(1.10 \%)$

$2(1.10 \%)$

Prostatic abscess

$1(0.55 \%)$

Renal tumour

Scrotal abscess

Scrotal hematoma

$1(0.55 \%)$

Urethral fistula

Urethral stone

$1(0.55 \%)$

Urethral trauma

UTI

$1(0.55 \%)$

$1(0.55 \%)$

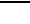

1 (0.55\%)

1 (0.55\%)

$1(0.55 \%)$

$1(0.55 \%)$

1 (0.55\%)

$1(0.55 \%)$

$1(0.55 \%)$

1 (0.55\%)

(0.55\%)

(0.55\%)

$1(0.55 \%)$

Total

$182(100.00 \%)$ 
Table 2.2. Procedures performed for patients having urologic surgery

\begin{tabular}{|c|c|c|}
\hline Procedure & n (\%) & Surgical assist \\
\hline Cystoscopy & $30(16.48 \%)$ & \\
\hline VIU & $26(14.29 \%)$ & $\begin{array}{l}8 \text { cases performed with urologists from } \\
\text { Canada }\end{array}$ \\
\hline Hydrocelectomy & $17(9.34 \%)$ & \\
\hline Simple prostatectomy & $15(8.24 \%)$ & \\
\hline Urethral Dilatation & $14(7.69 \%)$ & \\
\hline TURP & $12(6.59 \%)$ & $\begin{array}{l}11 \text { cases performed with urologist from } \\
\text { Germany / Canada }\end{array}$ \\
\hline Debridement for Fournier’s & $10(5.49 \%)$ & \\
\hline $\begin{array}{l}\text { Orchiectomy for } \\
\text { trauma/cryptorchidism }\end{array}$ & $10(5.49 \%)$ & \\
\hline $\begin{array}{l}\text { Bilateral orchiectomy for prostate } \\
\text { cancer }\end{array}$ & $9(4.95 \%)$ & \\
\hline Cystectomy with ileal conduit & $4(2.20 \%)$ & $\begin{array}{l}3 \text { cases performed in conjunction with } \mathrm{ZCH} \\
\text { general surgeon }\end{array}$ \\
\hline Open cystolithotomy & $4(2.20 \%)$ & \\
\hline TURBT & $4(2.20 \%)$ & Visiting urologists from Canada \\
\hline Circumcision & $3(1.65 \%)$ & \\
\hline Hypospadias repair & $3(1.65 \%)$ & Visiting urologist from Germany \\
\hline TURBN & $3(1.65 \%)$ & 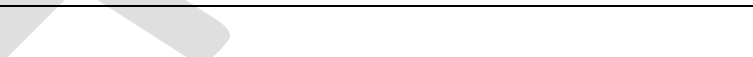 \\
\hline $\begin{array}{l}\text { Orchidopexy for undescended } \\
\text { testis }\end{array}$ & $2(1.10 \%)$ & Visiting urologists from Germany / Canada \\
\hline Partial cystectomy & $2(1.10 \%)$ & \\
\hline Shunt for priapism & $2(1.10 \%)$ & \\
\hline Suprapubic catheter & $2(1.10 \%)$ & \\
\hline Scrotal hematoma evacuation & $1(0.55 \%)$ & \\
\hline Open bladder repair for perforation & $1(0.55 \%)$ & \\
\hline Partial penectomy & $1(0.55 \%)$ & Visiting urologist from Germany \\
\hline Percutaneous nephrostomy & $1(0.55 \%)$ & \\
\hline Primary urethral realignment & $1(0.55 \%)$ & \\
\hline Paraphimosis reduction & $1(0.55 \%)$ & \\
\hline Open radical nephrectomy & $1(0.55 \%)$ & Performed by ZCH general surgeon \\
\hline Renal stone removal & $1(0.55 \%)$ & \\
\hline Total penectomy & $1(0.55 \%)$ & Visiting urologist from Germany \\
\hline Urethrocutaneostomy & $1(0.55 \%)$ & \\
\hline Total & $\begin{array}{c}182 \\
(100.00 \%)\end{array}$ & \\
\hline
\end{tabular}


TURP: transurethral resection of the prostate; TURBT: transurethral resection of the bladder tumor; TURBN: transurethral resection of the bladder neck; VIU: visual internal urethrotomy. Table 2.3. Anesthesia selection for patients undergoing urologic surgery

\begin{tabular}{|l|c|}
\hline Procedure & n (\%) \\
\hline Spinal & $125(68.68 \%)$ \\
\hline GA & $29(15.93 \%)$ \\
\hline Local & $28(15.38 \%)$ \\
\hline Total & $182(100.00 \%)$ \\
\hline
\end{tabular}

GA: general anesthetic. 